\title{
Molecular pathway activation features linked with transition from normal skin to primary and metastatic melanomas in human
}

\author{
Denis Shepelin ${ }^{1,2}$, Mikhail Korzinkin ${ }^{1,3}$, Anna Vanyushina ${ }^{4}$, Alexander Aliper ${ }^{4}$, \\ Nicolas Borisov ${ }^{3,5}$, Raif Vasilov5, Nikolay Zhukov ${ }^{3,6}$, Dmitry Sokov7, Vladimir \\ Prassolov $^{8}$, Nurshat Gaifullin ${ }^{9}$, Alex Zhavoronkov ${ }^{10}$, Bhupinder Bhullar ${ }^{11}$, Anton \\ Buzdin 1,4,5 \\ ${ }^{1}$ Pathway Pharmaceuticals, Wan Chai, Hong Kong, Hong Kong SAR \\ ${ }^{2}$ Group for Genomic Analysis of Cell Signaling Systems, Shemyakin-Ovchinnikov Institute of Bioorganic Chemistry, Moscow, \\ Russia \\ ${ }^{3}$ First Oncology Research and Advisory Center, Moscow, Russia \\ ${ }^{4}$ Laboratory of Bioinformatics, D. Rogachyov Federal Research Center of Pediatric Hematology, Oncology and Immunology, \\ Moscow, Russia \\ ${ }^{5}$ National Research Centre "Kurchatov Institute", Centre for Convergence of Nano-, Bio-, Information and Cognitive Sciences \\ and Technologies, Moscow, Russia \\ ${ }^{6}$ Pirogov Russian National Research Medical University, Department of Oncology, Hematology and Radiotherapy, Moscow, \\ Russia \\ ${ }^{7}$ Moscow 1st Oncological Hospital, Moscow Russia \\ ${ }^{8}$ Engelhardt Institute of Molecular Biology, Russian Academy of Sciences, Mosow, Russia \\ ${ }^{9}$ Moscow State University, Faculty of Fundamental Medicine, Moscow, Russia \\ ${ }^{10}$ Insilico Medicine, Inc, ETC, Johns Hopkins University, Baltimore, MD, USA \\ ${ }^{11}$ Novartis Institute for Biomedical Research, Basel, Switzerland
}

Correspondence to: Anton Buzdin, e-mail: buzdin@ponkc.com

Keywords: transition from nevus to primary and metastatic melanoma, OncoFinder, intracellular molecular networks, metabolic and signaling pathways, machine learning algorithms

Received: January 17, 2015

Accepted: November 11, 2015

Published: November 26, 2015

\section{ABSTRACT}

Melanoma is the most aggressive and dangerous type of skin cancer, but its molecular mechanisms remain largely unclear. For transcriptomic data of $\mathbf{4 7 8}$ primary and metastatic melanoma, nevi and normal skin samples, we performed highthroughput analysis of intracellular molecular networks including 592 signaling and metabolic pathways. We showed that at the molecular pathway level, the formation of nevi largely resembles transition from normal skin to primary melanoma. Using a combination of bioinformatic machine learning algorithms, we identified 44 characteristic signaling and metabolic pathways connected with the formation of nevi, development of primary melanoma, and its metastases. We created a model describing formation and progression of melanoma at the level of molecular pathway activation. We discovered six novel associations between activation of metabolic molecular pathways and progression of melanoma: for allopregnanolone biosynthesis, L-carnitine biosynthesis, zymosterol biosynthesis (inhibited in melanoma), fructose 2, 6-bisphosphate synthesis and dephosphorylation, resolvin D biosynthesis (activated in melanoma), D-myo-inositol hexakisphosphate biosynthesis (activated in primary, inhibited in metastatic melanoma). Finally, we discovered fourteen tightly coordinated functional clusters of molecular pathways. This study helps to decode molecular mechanisms underlying the development of melanoma. 


\section{INTRODUCTION}

Melanoma is a type of skin cancer formed from melanocytes, skin cells that produce the pigment melanin. Treatment of primary melanoma includes surgical removal, and in the case of early diagnosis, the US survival rate reaches $91 \%$. However, melanomas are very active in forming metastases, and if not diagnosed at the early stage, the survival prognosis is poor [1]. Melanoma accounts for $75 \%$ of deaths related to skin cancer [1]. In 2012, melanoma occurred in 232,000 patients and resulted in 55,000 deaths worldwide [2]. Development of melanomas is commonly caused by mutations from UV linked DNA damage [3] and by inherited genetic factors like highly penetrant loss-of-function mutations in tumor suppressor genes $C D K N 2 A$ and $X P[4,5]$. About $40 \%$ of human melanomas contain activating mutations of the B-Raf protein, resulting in constitutive signaling through the Raf to MAP kinases growth signaling pathways [6]. The presence of multiple melanocytic nevi, a genetic trait compounded by sun exposure, also increases the risk of developing melanoma, although the transition from benign nevi to melanoma does not usually occur and what triggers this change is unknown.

Melanoma cells are characterized by a high mutation rate. Genome-wide sequencing of twenty-five human melanomas identified $\sim 100$ structural rearrangements and $\sim 80,000$ mutated bases per genome [7]. This is roughly 1100 -times higher than the background mutation frequency in a normal human genome replicated between generations [8].

The molecular mechanisms of developing melanoma may be quite distinct. For example, UV irradiation causes keratinocytes to increase expression of multifunctional protein $\mathrm{p} 53$, which, by acting as a transcriptional factor, increases production of melanocyte-stimulating hormone (MSH) by these cells [9]. Secreted MSH molecules bind to melanocortin 1 receptors (MC1R) on the surface of melanocytes, which, in turn, promote the internal adenylate cyclase cascade and activate the CREB pathway, thus resulting in the activation of transcriptional factor MITF [10]. MITF, in turn, transactivates expression of p16 and Bcl2 proteins, which promote survival of melanocytes [11].

Alternatively, B-Raf, and its downstream signaling pathway through MAP kinases, directly promotes cell proliferation leading in melanomas, as evidenced by the positive clinical trials for B-Raf inhibitor drugs Dabrafenib and Vemurafenib $[13,14]$.

Another feature of invasive and metastatic melanoma cells is their ability to suppress the immune system, e.g. by overproducing CTLA-4 protein receptor, which inactivates T-cells [12]. Targeting this protein by the recently developed anticancer drug Ipilimumab showed enhanced survival for the advanced melanoma patients [13].

To learn more about the mechanisms that induce melanoma and cause it to progress, we performed highthroughput analysis of melanoma-related intracellular molecular networks including 592 signaling and metabolic pathways. We profiled a total of 478 transcriptomes corresponding to primary and metastatic melanoma, nevi and normal tissue samples. Using a combination of statistics and machine learning algorithms, we found characteristic sets of signaling and metabolic pathways activated or repressed during the development of primary melanoma from normal skin and also during its further progression to the metastatic state. We provide evidence that, at the molecular pathway level, formation of nevi clearly resembles the transitional state from normal skin to primary melanoma. For each stage of skin-to-melanoma transition, we identified characteristic molecular pathways, many of which are novel associations. Using bioinformatics analysis combined with various statistics and machine learning algorithms, we then created a stable model describing formation and progression of melanoma at the level of molecular pathway activation. Understanding the molecular mechanisms of melanoma development will be key in developing new treatment strategies.

\section{RESULTS AND DISCUSSION}

\section{Bioinformatics tool for the analysis of intracellular signaling and metabolic pathways}

We processed transcriptomic data from primary and metastatic melanoma, nevi, and reference normal samples to establish pathway activation strength (PAS) profiles corresponding to signaling and metabolic intracellular molecular pathways. Several approaches were published previously by us and others to measure PAS based on large scale gene expression data, either transcriptomic or proteomic. Khatri et al. [14] classified those methods into three major groups: Over-Representation Analysis (ORA), Functional Class Scoring (FCS) and Pathway Topology (PT)-based approaches. ORA-based methods calculate whether the pathway is significantly enriched with differentially expressed genes [15]. These methods have many limitations, as they ignore all non-differentially expressed genes and do not account for many gene-specific characteristics. FCS-based approaches partially tackle aforementioned limitations by calculating fold changebased scores for each gene and then combining them into a single pathway enrichment score [16]. PT-based analysis also takes into account topological characteristics of each given pathway, assigning additional weights to the genes (for a review, see [17]). To account for gene expression variability within a pathway, a set of differential variability methods has been developed [18]. Differential variability analysis determines a group of genes with a significant change in variance of gene expression between case and control groups [19]. This approach was further extended and applied on the pathway level [20].

Recently, we developed a new biomathematical method for pathway analysis, termed OncoFinder [21]. 
Based on kinetic models that use the "low-level" approach of mass action law, OncoFinder performs quantitative and qualitative enrichment analysis of the signaling pathways. For each sample investigated, it performs a casecontrol pairwise comparison and calculates the Pathway Activation Strength (PAS), a value which serves as a qualitative measure of pathway activation. Unlike most other methods, this approach determines if the signaling pathway is significantly up- or down-regulated compared to the reference. Negative and positive overall PAS values correspond to an inhibited or activated state of signaling pathway [21].

OncoFinder is also, to our knowledge, a unique PAS calculating method, which was reported to provide output data with significantly reduced noise introduced by the experimental transcriptome profiling systems [22]. This method was shown to be efficient in finding new cancer biomarkers, more stable than individual gene expression patterns [23]. Since its development, Oncofinder has been applied to the analysis of transcriptomes of various conditions, including leukemia and solid cancers [24-26], Hutchinson Gilford Disease [27] and Age-Related Macular Degeneration Disease [28].

Here, we updated the OncoFinder algorithm and the corresponding databases, which were originally developed to analyze only intracellular signaling pathways, to a new version supporting the analysis of both signaling and metabolic pathways. To build the internal interactions database for metabolic pathways, we used the publicly available HumanCyc database (www.humancyc.org). The resulting database used in this study contained 271 signaling and 321 metabolic intracellular pathways (Supplementary Dataset S1).

\section{Intracellular signaling and metabolic pathway activation profiles}

In this study, we profiled a group of 478 human transcriptomes consisting of 132 human primary melanoma, 222 metastatic melanoma, 103 normal skin and 21 nevi samples (Table 1).

The normalized gene expression data were next processed using the OncoFinder algorithm to establish pathway activation strength (PAS) profiles. The complete PAS data are shown on Supplementary Dataset S2. To assess the functional relations between the investigated groups of samples, we built hierarchical clustering heatmaps with Ward method using Euclidean distance for all samples and all investigated molecular pathways (Figure 1). We observed rather uncertain clustering features hardly distinguishing between the four sample classes. To increase the resolution of clustering methods and to identify features that distinguish the above functional groups, we applied a selection of machine learning classifier algorithms.

\section{Enhanced sampling classification with machine learning algorithms}

We used several different machine classifiers, including Random Forest (RF) Support Vector Machines (SVM) with Linear and Radial kernels, Partial Least Squares (PLS) and Generalized linear regression with Glmnet regularization. Prior to classification, we filtered for small deviation and collinearity to prevent using two highly correlated variables when one would suffice. Such approaches allowed us to achieve $\sim 0.94$ average balanced accuracy of a 4-class problem (classification into four groups: Skin, Nevi, Primary and Metastatic melanoma) using only metabolic pathways (Table 2 ) and $\sim 0.94$ average balanced accuracy using only signaling pathways (Table 3 ). In accordance with their vague transitional state, the most difficult group for all the classifiers used were nevi, for which the classifiers showed lowest combinations of sensitivity (0.4-0.8) and balanced accuracy (0.7-0.9) (Tables 2-3). Full statistical comparison of different classifiers are shown on Supplementary Dataset S3. Groups other than nevi formed significantly more clear-cut clusters, which corresponded to their physiologically distinct states. Overall, the SVM family classifiers showed the best results compared to other models.

\section{Identification of top pathways discriminating transition from skin to nevi and melanoma}

To further analyze progression of melanoma and relevant molecular events at the level of pathway activation, we used information of variable importance during the process of classification. For each statistical model, we identified the top 30 metabolic and top 30 signaling pathways, distinguishing the two classes using the "varImp" function from "caret" package, which unifies different techniques of measuring importance between different models (Supplementary Dataset S3). Next, the top pathways were intersected and a list of consensus pathways was established (Tables 4-5). The consensus records included 25 metabolic and 19 signaling pathways for two different models of melanoma development, the first occurring via transitional state of the nevus (Skin $\rightarrow$ Nevus $\rightarrow$ Melanoma) and the second not involving nevus (Skin $\rightarrow$ Primary Melanoma $\rightarrow$ Metastatic Melanoma). To test the classification power of these top pathways, we built a new hierarchical clustering heatmap with the Ward method, using Euclidean distance for all samples and top investigated molecular pathways with supporting Principal Components Analysis (PCA) projections plots (Figure 2). These top pathways enabled significantly better discrimination between the groups, as evidenced by PCA projections plots for all pathways (Figure 2A) compared to plots for the selected top pathways (Figure 2B). Next, we used these top pathways in the same 4-type prediction model as before. Results for best model 


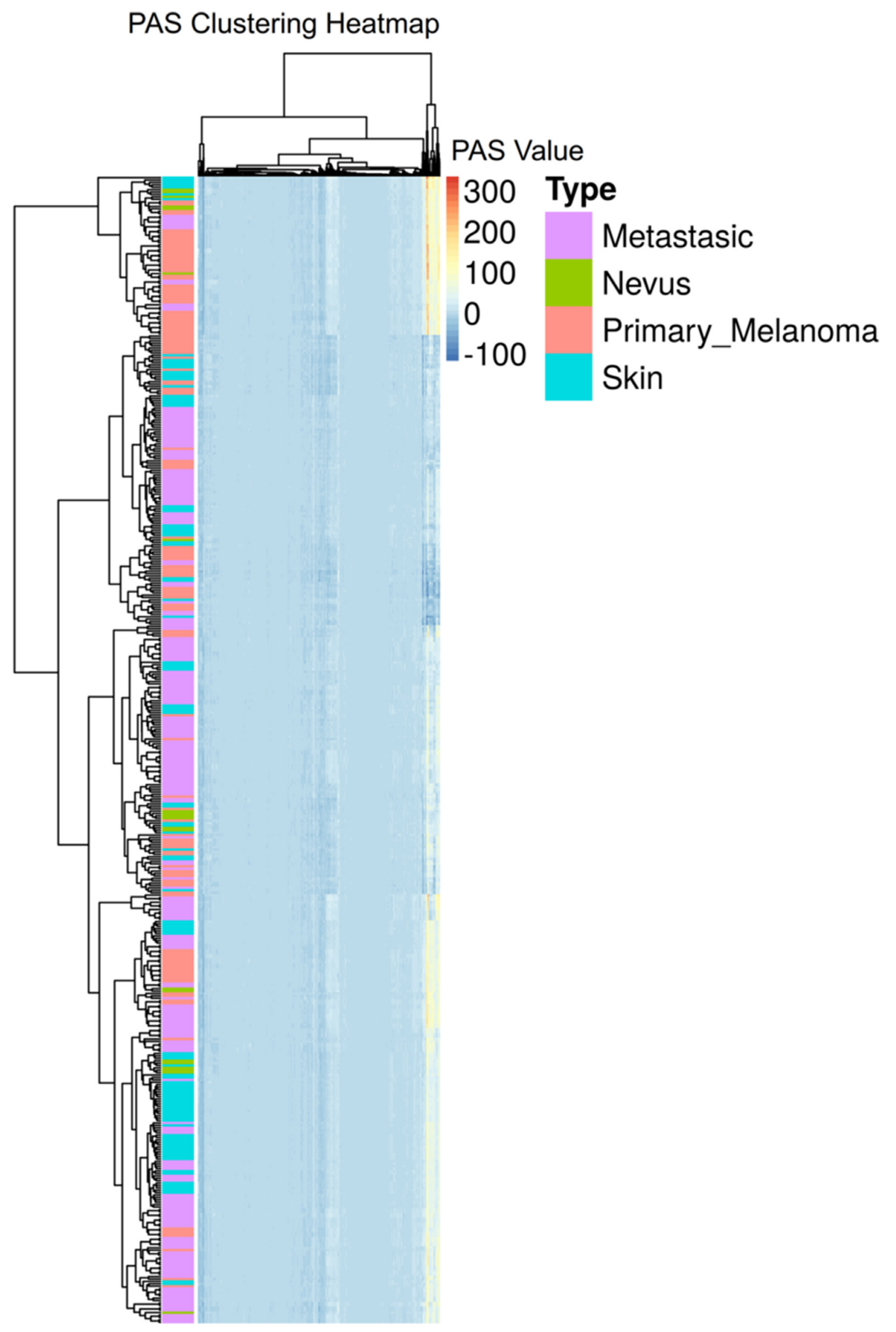

Figure 1: Hierarchical clustering heatmap of all samples and all molecular pathways under investigation. 
Table 1: Summary of transcriptomic datasets used in this study

\begin{tabular}{|c|c|c|c|c|c|}
\hline Dataset ID & Experimental platform & Skin samples & Nevus & Primary melanoma & $\begin{array}{l}\text { Metastatic } \\
\text { melanoma }\end{array}$ \\
\hline GSE 7553 & GPL570 & 5 & 0 & 14 & 40 \\
\hline GSE 53223 & GPL570 & 6 & 12 & 0 & 0 \\
\hline GSE 46517 & GPL96 & 8 & 9 & 31 & 52 \\
\hline GSE 39612 & GPL570 & 64 & 0 & 0 & 0 \\
\hline GSE 31879 & GPL570 & 4 & 0 & 10 & 0 \\
\hline GSE 23376 & GPL570 & 0 & 0 & 0 & 22 \\
\hline GSE 19234 & GPL570 & 0 & 0 & 0 & 44 \\
\hline GSE 15605 & GPL570 & 16 & 0 & 46 & 12 \\
\hline GSE 8401 & GPL96 & 0 & 0 & 31 & 52 \\
\hline
\end{tabular}

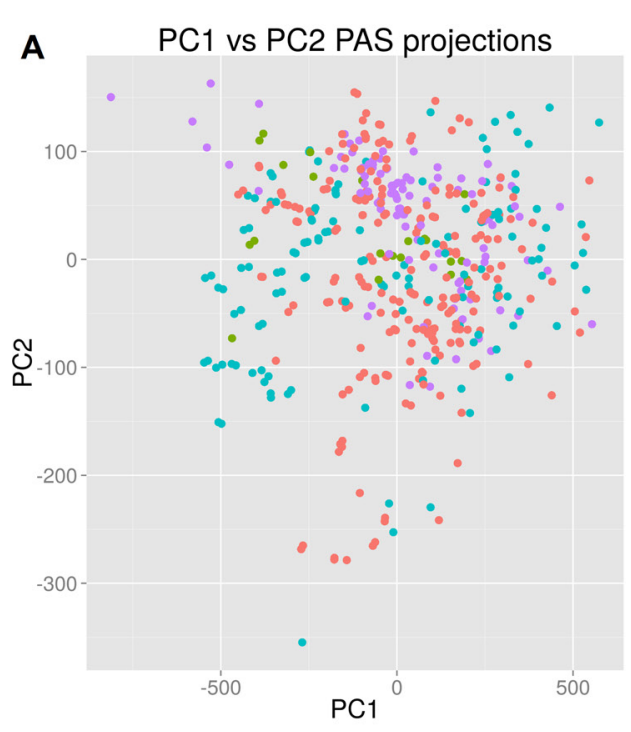

B

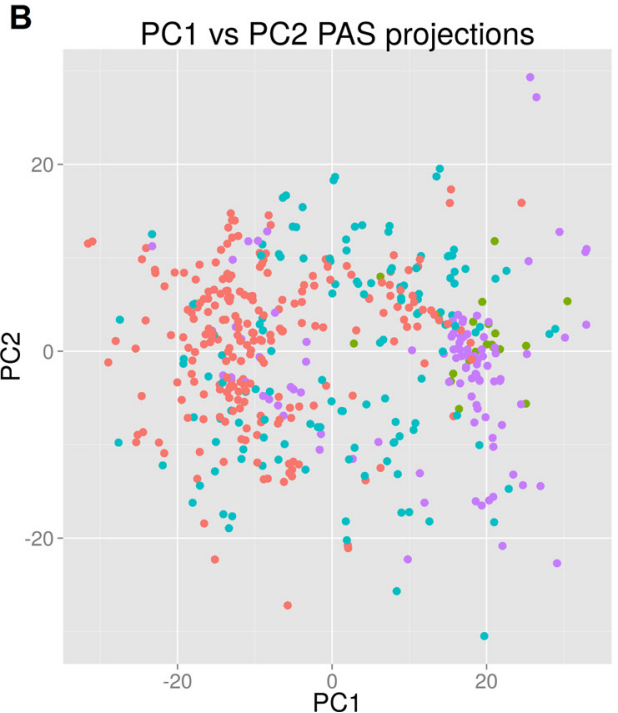

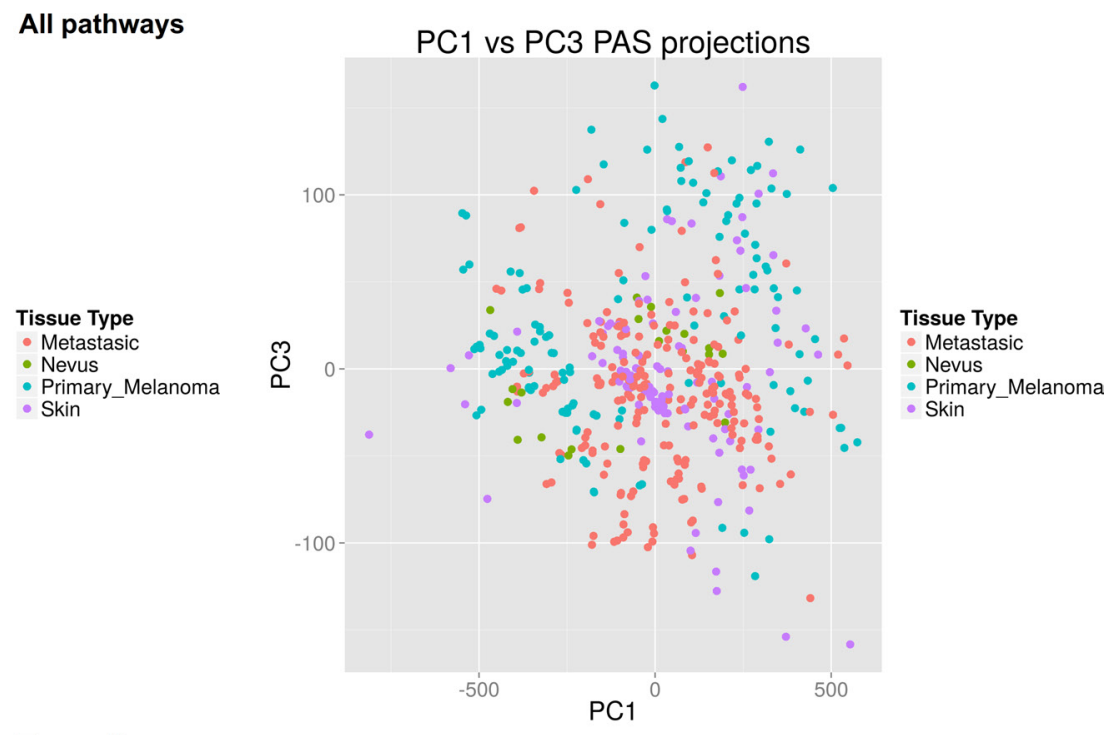

Top pathways

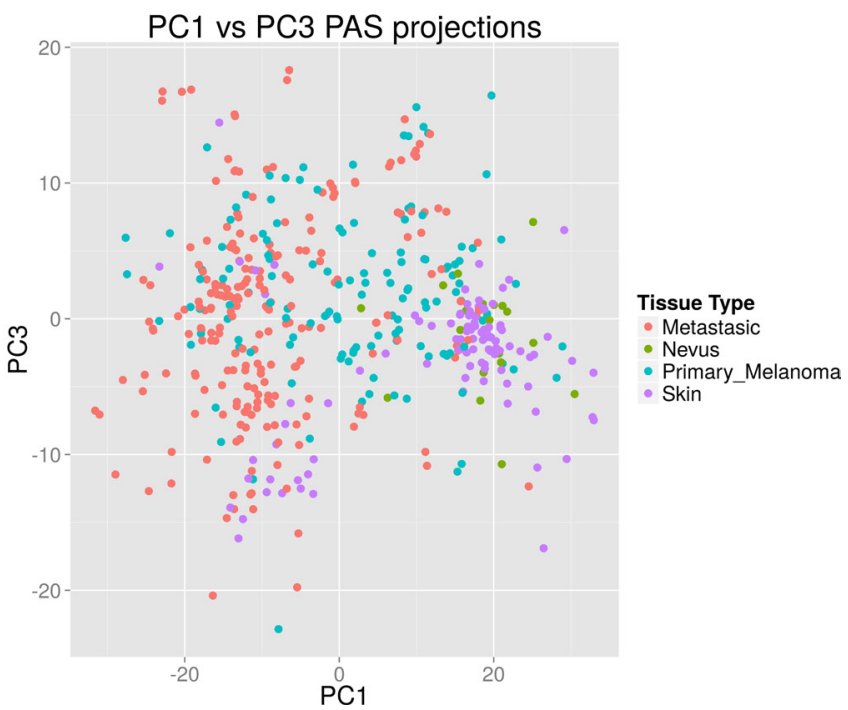

Figure 2: Scatterplots for principal component analysis. (A) Results built for all metabolic and signaling pathways. (B) Results built for top characteristic metabolic and signaling pathways. 
Table 2: SVM with Radial kernel classification report based on metabolic pathways

\begin{tabular}{|l|c|c|c|}
\hline \multicolumn{1}{c|}{ SVM Radial } & Sensitivity & Specificity & Balanced accuracy \\
\hline Metastatic Melanoma & 0.927 & 0.952 & 0.94 \\
\hline Nevus & 0.8 & 0.991 & 0.896 \\
\hline Primary Melanoma & 0.909 & 0.965 & 0.937 \\
\hline Skin & 1 & 0.989 & 0.995 \\
\hline
\end{tabular}

Average balanced accuracy 0,941 .

Table 3: SVM with Radial kernel classification report based on signaling pathways

\begin{tabular}{|l|c|c|c|}
\hline \multicolumn{1}{c}{ SVM Radial } & Sensitivity & Specificity & Balanced accuracy \\
\hline Metastatic Melanoma & 0.909 & 0.937 & 0.923 \\
\hline Nevus & 1 & 0.973 & 0.987 \\
\hline Primary Melanoma & 0.879 & 0.941 & 0.91 \\
\hline Skin & 0.88 & 1 & 0.94 \\
\hline
\end{tabular}

Average balanced accuracy 0, 939 .

(SVM Linear model) confirmed adequacy of the classifier pathway selection and showed an averaged balanced accuracy of $\sim 0.93$, very close to the model with full pathways (Table 6, Supplementary Dataset S4).

On the heatmap and PCA projection plots, the samples corresponding to nevi formed a cloudy group and clustered either with each other or diffusely between primary melanoma and normal skin samples. In agreement with previous reports, this suggests that nevi form a complicated group of highly variable samples, which frequently correspond to the intermediate state between normal skin and primary melanoma [29]. The top classifier elements included 25 metabolic and 19 signaling pathways. For all of these signaling pathways, association with melanoma was reported previously in the literature. However, for the metabolic pathways, this was not the case, and previous reports on the association with melanoma were not found for the following (Table 4): Allopregnanolone biosynthesis, L-carnitine biosynthesis, Zymosterol biosynthesis (inhibited in melanoma), D-myoinositol hexakisphosphate biosynthesis (activated in primary, inhibited in metastatic melanoma), Fructose 2, 6-bisphosphate synthesis and dephosphorylation, Resolvin $\mathrm{D}$ biosynthesis (activated in melanoma). Thus, we report here six novel associations between activation of metabolic molecular pathways and progression of melanoma.

\section{Functional significance of characteristic molecular pathways}

We observed a number of molecular pathway features distinguishing the transition from normal skin to either nevi or primary and metastatic melanomas (Tables 4, 5).
In melanomas, we identified several characteristically activated tumor suppressor pathways, like Fas signaling, a branch of ATM signaling leading to apoptosis and senescence, and the DDR pathway, leading to apoptosis. However, caspase cascade members were strongly inhibited in melanomas, which suggests that cancer cells efficiently escape cell death initiated at the upstream stages. This may be achieved by degradation of tumor suppressor proteins using proteasome-linked mechanisms (activated in both types of melanoma), and by activating cell survival and proliferation pathways like branches of CD40 signaling, HGF signaling and HIF1-Alpha pathway (activated in melanomas). Interestingly, the BRCA1 pathway dealing with DNA repair was strongly stimulated in melanomas, which may contribute to the relative inefficiency of radiation therapy treatment of metastatic melanomas.

Actively replicating cancer cells are faced with a lack of oxygen (hypoxia) and thus have to switch their energetic balance from oxidative phosphorylation to glycolysis. Glycolysis, which is based on anaerobic conversion of glucose to lactate, becomes the major source of ATP and NADH in the cells. This phenomenon, known as Warburg effect, is characteristic for most cancer cells [30]. Glycolysis is also the major source of substrates for basic biosynthetic pathways, e.g. those dealing with building ribonucleotides and amino acids [31]. For both nevi and melanomas, we observed increased expression of mannose-6-phosphate isomerase, an enzyme that makes it possible to utilize the sugar mannose as substrate for glycolysis. This, in turn, may intensify production of ATP by the transformed cells.

On the other hand, nevi-independent transformation to melanomas was characterized by gradual decrease of the nitric oxide (NO) biosynthesis pathway. NO is a 
Table 4: Top metabolic pathways implicated in progression of melanoma

\begin{tabular}{|c|c|c|c|c|c|}
\hline Pathway & $\begin{array}{l}\text { Nevus vs } \\
\text { Skin }\end{array}$ & $\begin{array}{l}\text { Pr. Mel } \\
\text { vs Skin }\end{array}$ & $\begin{array}{l}\text { Met. Mel } \\
\text { vs Skin }\end{array}$ & $\begin{array}{l}\text { Met.Mel } \\
\text { vs Pr.Mel }\end{array}$ & $\begin{array}{l}\text { Primary } \\
\text { vs Nevus }\end{array}$ \\
\hline allopregnanolone biosynthesis & UP & DOWN & DOWN & DOWN & DOWN \\
\hline citrulline-nitric oxide cycle & UP & DOWN & DOWN & DOWN & DOWN \\
\hline dTMP ide novoi biosynthesis mitochondrial & DOWN & UP & UP & UP & UP \\
\hline L-carnitine biosynthesis & UP & DOWN & DOWN & DOWN & DOWN \\
\hline 5-aminoimidazole ribonucleotide biosynthesis & DOWN & UP & UP & UP & UP \\
\hline eumelanin biosynthesis & UP & UP & UP & DOWN & DOWN \\
\hline putrescine biosynthesis II & DOWN & DOWN & UP & UP & UP \\
\hline pyrimidine deoxyribonucleosides salvage & DOWN & UP & UP & UP & UP \\
\hline spermine and spermidine degradation I & UP & DOWN & DOWN & DOWN & DOWN \\
\hline superpathway of tryptophan utilization & UP & DOWN & DOWN & UP & DOWN \\
\hline tryptophan degradation X mammalian via tryptamine & UP & DOWN & DOWN & DOWN & DOWN \\
\hline $\begin{array}{l}\text { 1D-imyoi-inositol hexakisphosphate biosynthesis V from } \\
\text { Ins134P3 }\end{array}$ & UP & UP & DOWN & DOWN & UP \\
\hline D-mannose degradation & UP & UP & UP & UP & DOWN \\
\hline fructose 26-bisphosphate synthesis, dephosphorylation & UP & UP & UP & DOWN & DOWN \\
\hline histamine biosynthesis & UP & DOWN & DOWN & DOWN & DOWN \\
\hline inosine-5-phosphate biosynthesis & UP & UP & UP & UP & DOWN \\
\hline melatonin degradation II & UP & DOWN & DOWN & DOWN & DOWN \\
\hline pyrimidine deoxyribonucleosides degradation & UP & UP & UP & DOWN & UP \\
\hline resolvin $\mathrm{D}$ biosynthesis & UP & UP & UP & DOWN & UP \\
\hline retinoate biosynthesis I & DOWN & DOWN & DOWN & UP & UP \\
\hline superpathway of steroid hormone biosynthesis & UP & DOWN & DOWN & DOWN & DOWN \\
\hline tRNA charging & UP & UP & UP & UP & UP \\
\hline UDP-N-acetyl-D-galactosamine biosynthesis II & UP & UP & UP & UP & DOWN \\
\hline valine degradation & DOWN & DOWN & DOWN & UP & DOWN \\
\hline zymosterol biosynthesis & UP & DOWN & DOWN & DOWN & DOWN \\
\hline
\end{tabular}

UP or DOWN indicates positive and negative difference between the state of interest (nevus, primary and metastatic melanoma) and skin in median PAS value, respectively.

known mediator of cancer aggressiveness. Mechanisms of its influence depend greatly on concentration and time of exposure. Low concentrations stimulate proliferation of cancer cells, whereas high levels of NO may cause antitumor effects [32]. Low levels $(<300 \mathrm{nM})$ promote cell survival and proliferation by activating mTOR, cyclic GMP signaling, by Akt phosphorylation and by stabilization of hypoxia-induced factor HIF-1 $\alpha$ [33]. At the same time, low NO activates glycolysis by stimulating AMP-protein kinases, by increasing concentrations of fructose-2, 6-bisphosphatase, and by promoting glucose uptake by the cells [34]. In contrast, increased levels of
NO (greater than $300 \mathrm{nM}$ ) promote activation of $\mathrm{p} 53$ signaling and related cytostatic and apoptotic effects linked with inactivation of ERK and Akt signaling [35].

We speculate that the apparently stimulated NO synthesis pathway in nevi may be one of the factors that stabilizes their condition and prevents cancer transformation. Activity of the enzymes involved in NO biosynthesis, like arginine succinate synthase (ASS) depends on the regulation by glucocorticoid hormones cAMP, glucagon and insulin. Reduced expression of ASS was previously reported in the literature for melanoma and other tumors [36]. Cancer cells frequently are unable to synthesize sufficient amounts of 
Table 5: Top signaling pathways implicated in progression of melanoma

\begin{tabular}{|c|c|c|c|c|c|}
\hline Pathway & $\begin{array}{c}\text { Nevus vs } \\
\text { Skin }\end{array}$ & $\begin{array}{l}\text { Pr. Mel vs } \\
\text { Skin }\end{array}$ & $\begin{array}{l}\text { Met. Mel } \\
\text { vs Skin }\end{array}$ & $\begin{array}{c}\text { Met.Mel } \\
\text { vs Pr.Mel }\end{array}$ & $\begin{array}{c}\text { Pr. Mel vs } \\
\text { Nevus }\end{array}$ \\
\hline Fas Signaling Pathway (Negative) & DOWN & UP & UP & UP & UP \\
\hline cAMP Pathway (Glycolysis) & UP & DOWN & DOWN & UP & DOWN \\
\hline CD40 Pathway (Cell Survival) & UP & UP & UP & UP & UP \\
\hline AKT Pathway (Protein Synthesis) & UP & DOWN & DOWN & DOWN & DOWN \\
\hline ATM Pathway (Apoptosis, Senescense) & DOWN & UP & UP & UP & UP \\
\hline BRCA1 Main Pathway & UP & UP & UP & UP & UP \\
\hline cAMP Pathway (Endothelial Cell Regulation) & UP & DOWN & DOWN & DOWN & DOWN \\
\hline cAMP Pathway (Myocardial Contraction) & DOWN & DOWN & DOWN & DOWN & DOWN \\
\hline cAMP Pathway (Protein Retention) & DOWN & UP & UP & UP & UP \\
\hline Caspase Cascade (Apoptosis) & UP & DOWN & DOWN & DOWN & DOWN \\
\hline CD40 Pathway (IKBs Degradation) & UP & UP & UP & UP & UP \\
\hline DDR pathway Apoptosis & DOWN & UP & UP & UP & UP \\
\hline Glucocorticoid Receptor Pathway (Cell cycle arrest) & UP & DOWN & DOWN & DOWN & DOWN \\
\hline HGF Pathway (PKC pathway) & UP & UP & UP & UP & DOWN \\
\hline HIF1-Alpha Main Pathway & UP & UP & UP & UP & UP \\
\hline JNK Pathway (Insulin signaling) & UP & DOWN & DOWN & DOWN & DOWN \\
\hline mTOR Pathway (VEGF pathway) & DOWN & DOWN & UP & UP & DOWN \\
\hline PAK Pathway (Myosin Activation) & DOWN & DOWN & DOWN & DOWN & DOWN \\
\hline Ubiquitin Proteasome Pathway (Degraded Protein) & DOWN & UP & UP & UP & UP \\
\hline
\end{tabular}

UP or DOWN indicates positive and negative difference between the states of interest (nevus, primary and metastatic melanoma) and skin in median PAS value, respectively.

arginine and thus fully depend on the import of this amino acid from blood. It was shown previously that arginine deprivation in blood using the enzyme arginine deimynidase may result in cancer regression [36]. Moreover, arginine is tightly associated with the metabolism of polyamines in eukaryotic cells. Arginine is the precursor of ornithine, which is a substrate for further biosynthesis of polyamines [37]. Thus, reduction of arginine biosynthesis may cause altered concentrations and metabolism for putrescin, spermidine, spermine and other polyamines in melanoma. In this study, we show suppressed degradation of spermine and spermidine in both types of melanoma, which may help the cells to store the synthesized polyamines. The polyamines are major organic cations presenting in the eukaryotic cells. These molecules are absolutely necessary for cell growth and differentiation [38]. They can nonspecifically activate DNA replication, transcription and translation [39]. For example, increased concentrations of polyamines stimulate proliferation, increase synthesis of the external matrix proteins and enhance angiogenesis [40]. Ornitindecarboxylase (ODC) is the first enzyme in the pathway of polyamine synthesis. In transgenic mice, progression of melanoma depends on the biosynthesis of polyamines, particularly putrescin, as treatment with ODC inhibitors inhibits tumor growth and causes its rapid regression [41]. Moreover, inhibitors of polyamine synthesis enzymes efficiently decrease frequencies of spontaneous skin cancers caused by UV irradiation or induced by chemicals in different experimental models. In contrast, artificial induction of ODC activity causes a sequential increase in concentrations of polyamines followed by enhanced frequencies of skin cancers [41].

Furthermore, we observed sequential inhibition of a pathway of histamine biosynthesis in both types of melanoma. Histamine is a diamine molecule that has important neurotropic activities but also modulates immune response by increasing permeability of blood vessels to leukocytes and proteins [42]. In line with our observations, an antagonistic relationship was previously reported for polyamines and histamine because of partial inhibition of intracellular uptake of polyamines by the histamine [42]. 
Table 6: SVM with Linear kernel method classification report based on combination of top signaling and metabolic pathways

\begin{tabular}{|l|c|c|c|}
\hline \multicolumn{1}{c}{ SVM Linear } & Sensitivity & Specificity & Balanced accuracy \\
\hline Metastasic & 0.909 & 0.889 & 0.899 \\
\hline Nevus & 1 & 0.991 & 0.996 \\
\hline Primary Melanoma & 0.788 & 0.965 & 0.876 \\
\hline Skin & 0.96 & 0.978 & 0.969 \\
\hline
\end{tabular}

Average balanced accuracy 0, 935 .

Another feature distinguishing normal skin, nevi, and melanomas was the pathway of carnitine biosynthesis, apparently activated in nevi and inhibited in melanomas. Carnitine is a compound required for fatty acid metabolism in mammals. When entering the cell, free fatty acids are oxidized to form acil-CoA molecules, which are next transferred to the mitochondrial matrix using carnitinepalmitoyl transferase 1 (CPT1) and carnitine [43]. The CPT1-based transfer system is, therefore, directly linked with the biosynthesis of carnitine and a hypoxic state [44]. Carnitine is synthesized using two amino acid substrates, lysine and methionine, in a reaction catalyzed by trimethyl-lysine-dioxygenase (TMLH) [45]. The observed increase in carnitine biosynthesis regulation may be connected with the hypoxic conditions characteristic for primary and metastatic melanomas, both of which feature upregulation of the hypoxia-induced factor 1 (HIF1) pathway. The melanoma cells, therefore, may be deficient in their ability to use fatty acids as the substrate for oxidative phosphorylation; this deficiency may be an object of future molecular therapeutic approaches.

We also noticed decreased activation of the tryptophan degradation pathway in melanomas, which may lead to tryptophan accumulation in the cells. Tryptophan is the precursor of many signaling molecules, including melatonin and serotonin [46], and also of NAD and niacin. Congruently, we found that a pathway of melatonin degradation is decreased in both types of melanoma. Thus, regulation of melatonin and tryptophan degradation may be correlated in melanoma cells.

\section{Generalized molecular model of transition from normal skin to melanoma and nevi}

We found 25 metabolic and 19 signaling pathways that were good-quality characteristic discriminators between the classes of normal skin, nevus, primary melanoma and serotonin metastatic melanoma (Tables 4, 5). We considered two general models of melanoma formation and transformation including transitions (i) Skin $\rightarrow$ Nevus $\rightarrow$ Prtimary melanoma $\rightarrow$ Metastatic melanoma) and nevus-independent model (ii) Skin $\rightarrow$ Primary Melanoma $\rightarrow$ Metastatic Melanoma (Figure 3). In both transition axes, HIF1-alpha and BRCA1 pathways were gradually increasing when moving from normal state to metastatic melanoma.
Transition from normal skin to nevi compared to primary melanoma was very peculiar because it included activation of histamine, allopregnanolone, and citrulline NO cycle biosynthesis pathways. Eumelanin biosynthesis and BRCA1, HIF1-alpha signaling pathways were also activated. Several pathways were also suppressed in nevi, in contrast to primary and metastatic melanomas; these included putrescin biosynthesis, valine degradation, and the senescence/apoptotic branch of the ATM pathway.

Transition from normal skin to primary melanoma was characterized by upregulation of the eumelanin biosynthesis pathway, BRCA1, HIF1-alpha pathways, senescence/apoptotic branch of the ATM pathway, cell death-promoting Fas signaling pathways, and the cell survival-promoting branch of the CD40 pathway. In turn, pathways of putrescine, histamine, allopregnanolone, steroid hormone and citrulline - NO cycle biosynthesis and of valine degradation were inhibited in primary melanoma compared to skin.

Transition from nevus to primary melanoma showed upregulation of the BRCA1, HIF1-alpha pathways, senescence/apoptotic branch of the ATM pathway, and putrescine biosynthesis pathway. Inhibited pathways were histamine, allopregnanolone, eumelanin biosynthesis and citrulline - NO cycle biosynthesis and of valine degradation.

Finally, transition from primary to metastatic melanoma comprised upregulation of BRCA1, HIF1alpha pathways, the senescence/apoptotic branch of the ATM pathway, putrescine biosynthesis, and valine degradation pathways. Inhibited pathways were histamine, allopregnanolone, eumelanin biosynthesis, and citrulline NO cycle biosynthesis. The complete list of characteristic pathways is shown on Tables 4 and 5.

\section{Epigenetic regulation of melanoma advancement}

The HIF1 pathway is specifically activated in the critical transitions from nevus to primary melanoma and, again to the more aggressive metastatic melanoma. HIF1alpha has a tight correlation to epigenetic mechanisms. It has been demonstrated that HIF1-alpha induces expression of histone demethylases, JARID1C and JMJD1A for example, in colorectal cancer cells, which directly promote malignant development by epigenetic mechanisms $[47,48]$. Since chromatin remodeling is associated with 
changes in gene expression, a closer look at the pathways regulated by HIF1-alpha, would help elucidate significant molecular pathways promoting the transitions to primary and metastatic melanoma, This does not preclude other epigenetic mechanisms that may be specific to the development of melanoma. For example, studies have shown the role of micro-RNA, miR-211 and miR-375, have tumor suppressive functions in melanocytes $[49,50]$. Silencing of both micro-RNA species have been observed in melanoma, and indeed, epigenetic downregulation of miR-375 locus was observed as one mechanism of this silencing [50]. Taken together, the role of epigenetics in melanoma needs to be investigated further, and using Pathway Activation Scoring, we may be able to delineate the relationships with the metabolic and signaling pathways that we have described above.

\section{Congruent activation of various sets of molecular pathways}

For the first time, we explored here the activity of 592 signaling and metabolic pathways using the hierarchical clustering assay. We applied the Weighted Correlation Network Analysis (WGCNA) method to identify similar regulation patterns between the molecular pathways. We found that molecular pathways form 14 distinguishable clusters, each characterized by concordant activation signatures of the enclosing pathways (Jaccard structural similarity index signatures are shown separately for each cluster on Supplementary Dataset S5). All pathways in clusters were filtered according to their paired and overall correlation coefficients. All molecular pathways from each cluster are listed in Supplementary Dataset S6.

In some instances, congruent activation for the pathways forming the same clusters can be explained by the structural similarities between the cluster-forming

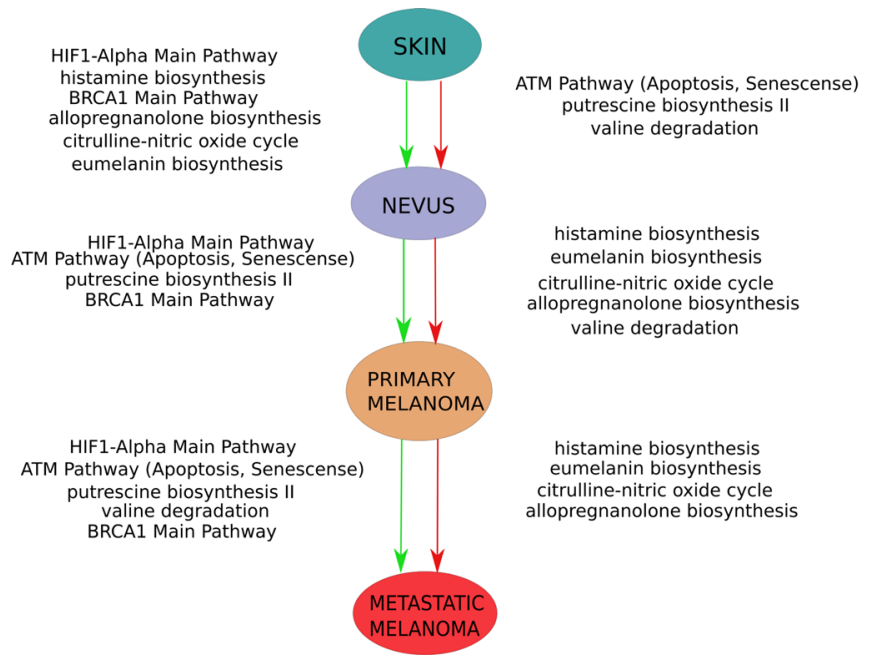

pathways - e.g., AKT Pathway (Caspase Cascade) and AKT Pathway (p73 Mediated Apoptosis) from cluster \#8, or p53 Signaling (Negative) Pathway (p53 Degradation) and Wnt Pathway (Ctnn-b Degradation) from cluster \#5 are highly similar in their gene product composition (Supplementary Dataset S5). However, for the majority (10 out of 14) of clusters, pathways were combined not due to similar gene content, but rather because of the true functional coordination between the cluster members (Supplementary Dataset S5). For example, for cluster 6 shown on Figure 4, most of the enclosing pathways have low structural similarity, but are at the same time strongly functionally coordinated, as reflected by low Jaccard indexes and high PAS correlation scores, respectively. This common regulation of various molecular pathways is a novel finding and will be analyzed in detail in further studies.

\section{CONCLUSIONS}

Here, we provide evidence that at the molecular pathway level, nevi largely correspond to a transitional state from normal skin to primary melanoma. We found 44 signaling and metabolic pathways connected with the formation of nevi and with the development of primary melanoma and its metastases. We created a stable model describing formation and progression of melanoma at the level of molecular pathway activation. Many of these pathways had never been previously associated with melanoma. We found six novel associations between activation of metabolic molecular pathways and progression of melanoma. Finally, we discovered fourteen tightly coordinated functional clusters of molecular signaling and metabolic pathways. This study helps to decode molecular mechanisms underlying development of melanoma.

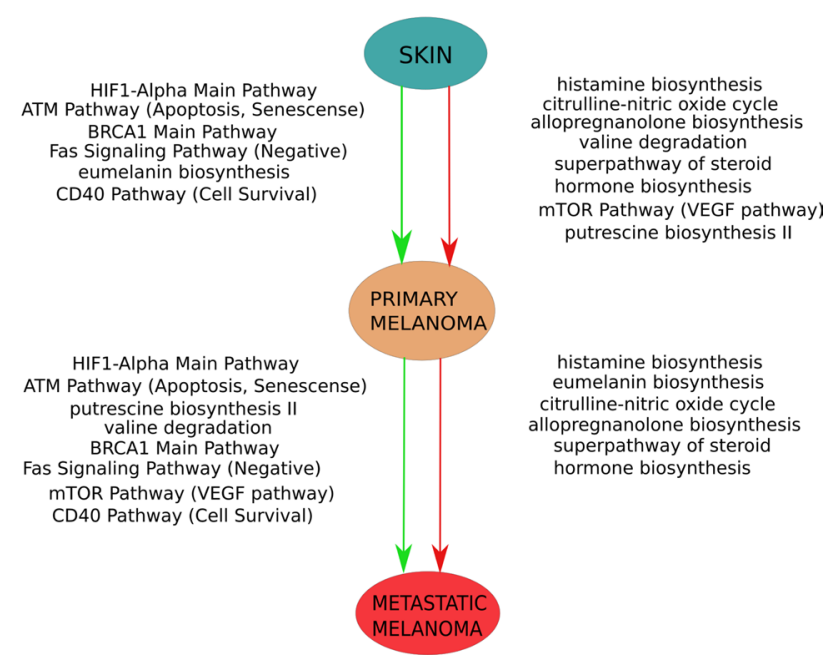

Figure 3: Schematic representation of two alternative models of melanoma progression built in this study. One model comprises transition from skin to primary melanoma versus "nevus" stage (left panel), the second - direct transition from skin to primary melanoma (right panel). Green arrows indicate activated molecular pathways, red arrows - suppressed pathways. 


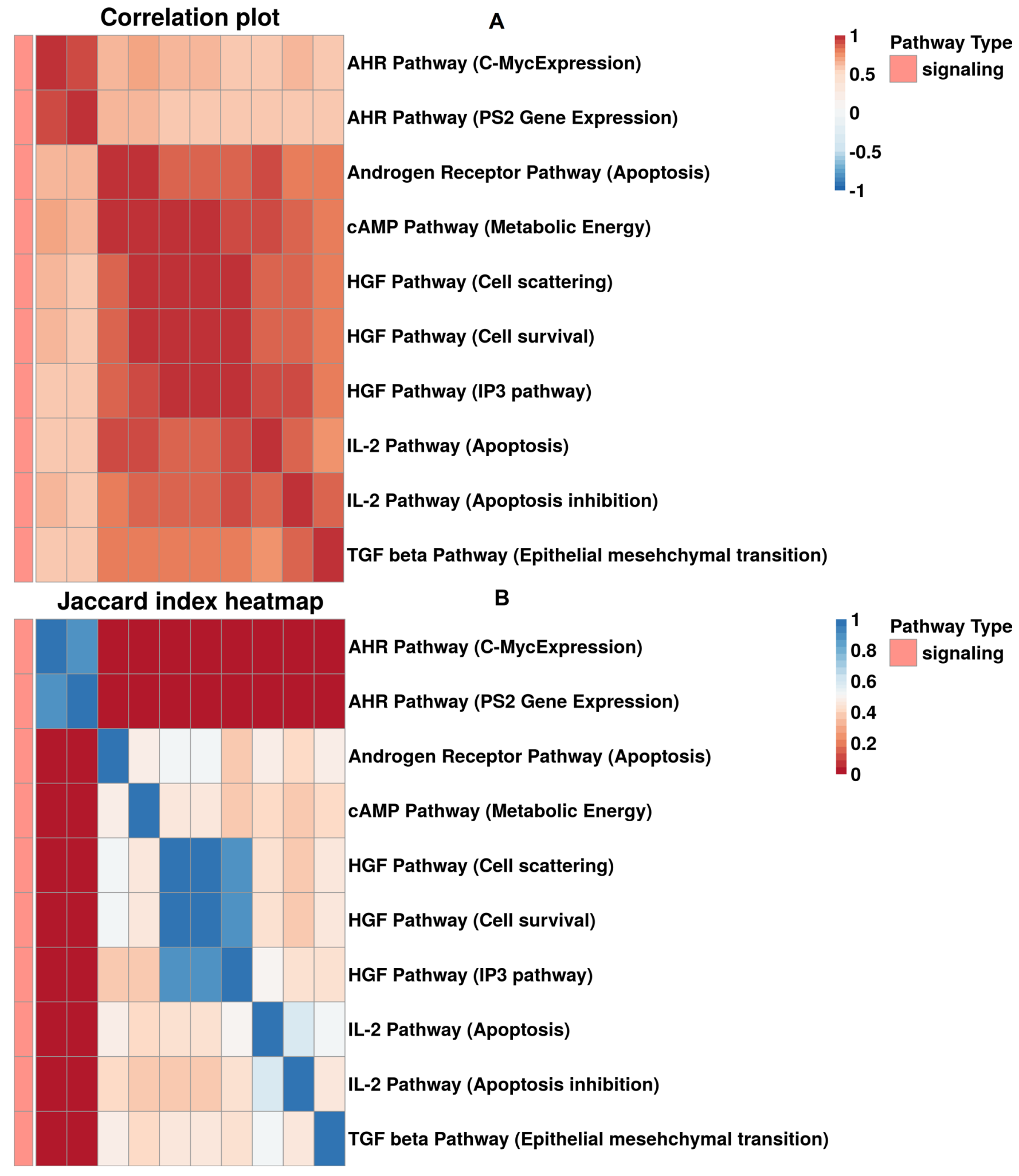

Figure 4: (A) Correlation plot built for cluster 6. (B) Heatmap of Jaccard gene intersection index between pathways in cluster 6. 


\section{MATERIALS AND METHODS}

\section{Transcriptomic datasets}

We obtained from NCBI GEO repository (www.ncbi. nlm.nih.gov/geo/) nine datasets containing transcriptomic information for human tissues related to melanoma: GSE7553, GSE53223, GSE46517, GSE39612, GSE31879, GSE23376, GSE19234, GSE15605 and GSE8401, and one dataset with the transcriptomic data for Agilent Universal Human RNA Reference (GSE3061). For all the datasets, experimental transcriptional profiling was performed using Affymetrix GPL90 and GPL570 platforms. The data from different datasets were quantile-normalized using «affy» package for R 3.1.1 for each platform, and afterwards combined together followed by the harmonization with the XPN algorithm for cross-platform normalization of samples [51]. Each sample under study, except Agilent Universal Human RNA Reference, was classified to one of the groups below: "Normal Skin", "Primary Melanoma", "Metastatic Melanoma" or "Nevi", according to dataset description. In total, we included 103 Skin, 21 Nevus, 132 Primary Melanoma, and 222 Metastatic Melanoma samples in our study.

\section{Functional annotation of gene expression data}

For the functional annotation of gene expression data at the molecular pathway level, we applied OncoFinder algorithm, recently published by Buzdin et al. [21]. It operates with calculation of the Pathway Activation Strength (PAS), a value which serves as a qualitative measure of a molecular pathway activation. The formula for PAS calculation accounts for gene expression data and for information on the protein interactions in a pathway, namely, individual protein activator or repressor roles in a pathway [24].

The positive value of PAS indicates abnormal activation of a signaling pathway, and the negative value - its repression. Here, the case-to-normal ratio, $\mathrm{CNR}_{\mathrm{n}}$, is the ratio of expression levels for a gene $n$ in the sample under investigation to the same average value for the control group of samples. In addition, for each CNR value, we applied multiplication to a Boolean flag of BTIF (beyond tolerance interval flag), which equals to 1 when the CNR value passed, and to 0 when CNR value did not pass both or either one of the two criteria of significantly differential expression: first, the expression level for the sample must fit outside of the tolerance interval for norms, with $p<0.05$, and second, the value of CNR must differ from 1 by at least 1.5-fold. For each sample, we obtained results for the 269 signaling and 363 metabolic pathways, in standard application using Agilent Universal Human RNA Reference samples as the norms. Alternatively, for some calculations, the norms were the normal human skin samples.

\section{Classification and feature selection}

We applied the following frequently used machine learning algorithms for classification of the samples SVM (Support Vector Machine) with linear and radial kernel ("e1071" package), Random Forest ("rf"), Lasso ("glmnet"), Partial Least Squares ("pls") and Boosted Logistical Regression ("caTools"), Naive Bayes Classifier ("klaR"). R package "caret" was employed to implement uniform interface for each of the above learning algorithms. To prevent overfitting, we used repeated 10-fold crossvalidation. Before training these classifiers, we performed different feature selections - features (PAS scores for different molecular pathways) with near zero variance were removed using the helper function from "caret" package termed "nearZeroVar", while collinear predictors were identified and removed with the function "findCorrelation" from "caret" package, with threshold setting 0.85 .

\section{Identification of top molecular pathways}

We applied a two-step scheme to identify the most relevant pathways for classification of the melanoma samples. At the first step, we used function "varImp" from the caret package to obtain the top 30 pathways for each classificator model. Next, we looked for the intersections among all the models. The second step was the KruskallWallis test, implemented in package "agricolae," and removal of pathways not significantly differentially regulated $(p>0.05)$ between at least two different classes in progressions Skin $\rightarrow$ Nevus $\rightarrow$ Primary Melanoma, and Skin $\rightarrow$ Primary Melanoma $\rightarrow$ Metastasic Melanoma.

\section{Clustering}

To explore subtypes of primary and metastasic melanoma, we applied the hierarchical clustering method "hclust" with Ward distance and cutted clustering dendrogram at height 2000. We used $\mathrm{R}$ package "WGCNA" to find clusters of correlating pathways in melanoma samples. Function "blockwiseModules" was used to create a network, with minimum of 5 pathways in subnetworks. A correlation diagram was built using function "pheatmap" from the package "pheatmap," sorted with respect to hierarchical clustering.

Each pathway in a cluster was filtered by correlation with other paths in the cluster - either with a mean modulus of correlation coefficient more than 0.7 , or with at least one coefficient more than 0.85 and not 1 (correlation with itself). For each cluster, we also built heatmaps of Jaccard coefficient of gene intersections.

\section{Heatmaps and statistical analysis}

We built our hierarchical clustering heatmaps using function "pheatmap" from "pheatmap" package; we also 
used this to create cluster images and correlational plots. PCA plots were made with help of "prcomp" function in R. Changes in pathway activation in different groups were measured by median change and $t$-test with multiple test correction.

\section{ACKNOWLEDGMENTS}

We would like to thank Dr. Leslie Jellen for help with editing of the manuscript.

\section{GRANT SUPPORT}

This work was supported by the Russian Science Foundation grant no. 14-14-01089 (for V. Prassolov and Anton Buzdin), by the Pathway Pharmaceuticals (HongKong) and First Oncology Research and Advisory Center (Russia) Joint Research Initiative (for Mikhail Korzinkin, Anna Vanyushina, Alexander Aliper, Nicolas Borisov, Nikolay Zhukov and Alex Zhavoronkov) and by the Program of the Presidium of the Russian Academy of Sciences "Dynamics and Conservation of Genomes" (for Denis Shepelin).

\section{CONFLICTS OF INTEREST}

The authors declare that they have no potential conflict of interests in relation to publishing this paper.

\section{REFERENCES}

1. Jerant AF, Johnson JT, Sheridan CD, Caffrey TJ. Early detection and treatment of skin cancer. Am Fam Physician. 2000; 62:357-68, 375-6, 381-2.

2. Stewart BW, Wild C. International Agency for Research on Cancer, World Health Organization, editors. World cancer report 2014. Lyon, France: International Agency for Research on Cancer. 2014; 630.

3. Ghissassi F El, Baan R, Straif K, Grosse Y, Secretan B, Bouvard V, Benbrahim-Tallaa L, Guha N, Freeman C, Galichet L, Cogliano V. WHO International Agency for Research on Cancer Monograph Working Group. A review of human carcinogens_-part D: radiation. Lancet Oncol. 2009 ; 10:751-2.

4. Greene MH. The genetics of hereditary melanoma and nevi. 1998 update. Cancer. 1999; 86:2464-77.

5. Halachmi S, Gilchrest BA. Update on genetic events in the pathogenesis of melanoma. Curr Opin Oncol. 2001; 13:129-36.

6. Davies MA, Samuels Y. Analysis of the genome to personalize therapy for melanoma. Oncogene. 2010; 29:5545-55.

7. Berger MF, Hodis E, Heffernan TP, Deribe YL, Lawrence MS, Protopopov A, Ivanova E, Watson IR, Nickerson E, Ghosh P, Zhang H, Zeid R, Ren X, et al.
Melanoma genome sequencing reveals frequent PREX2 mutations. Nature. 2012; 485:502-6.

8. Campbell CD, Chong JX, Malig M, Ko A, Dumont BL, Han L, Vives L, O'Roak BJ, Sudmant PH, Shendure J, Abney M, Ober C, Eichler EE. Estimating the human mutation rate using autozygosity in a founder population. Nat Genet. 2012; 44:1277-81.

9. Swope V, Alexander C, Starner R, Schwemberger S, Babcock G, Abdel-Malek ZA. Significance of the melanocortin 1 receptor in the DNA damage response of human melanocytes to ultraviolet radiation. Pigment Cell Melanoma Res. 2014; 27:601-10.

10. Rodríguez CI, Setaluri V. Cyclic AMP (cAMP) signaling in melanocytes and melanoma. Arch Biochem Biophys. 2014; 563:22-7.

11. Hartman ML, Czyz M. Pro-survival role of MITF in melanoma. J Invest Dermatol. 2015; 135:352-8.

12. Lee KM, Chuang E, Griffin M, Khattri R, Hong DK, Zhang W, Straus D, Samelson LE, Thompson CB, Bluestone JA. Molecular basis of T cell inactivation by CTLA-4. Science. 1998; 282:2263-6.

13. Ito A, Kondo S, Tada K, Kitano S. Clinical Development of Immune Checkpoint Inhibitors. Biomed Res Int. 2015; 2015:605478.

14. Khatri P, Sirota M, Butte AJ. Ten years of pathway analysis: current approaches and outstanding challenges. PLoS Comput Biol. 2012; 8:e1002375.

15. Khatri $\mathrm{P}$, Drăghici S. Ontological analysis of gene expression data: current tools, limitations, and open problems. Bioinformatics. 2005; 21:3587-95.

16. Tian L, Greenberg SA, Kong SW, Altschuler J, Kohane IS, Park PJ. Discovering statistically significant pathways in expression profiling studies. Proc Natl Acad Sci USA. 2005; 102:13544-9.

17. Mitrea C, Taghavi Z, Bokanizad B, Hanoudi S, Tagett R, Donato M, Voichiţa C, Drăghici S. Methods and approaches in the topology-based analysis of biological pathways. Front Physiol. 2013; 4:278.

18. Afsari B, Geman D, Fertig EJ. Learning dysregulated pathways in cancers from differential variability analysis. Cancer Inform. 2014; 13:61-7.

19. Ho JWK, Stefani M, Remedios CG dos, Charleston MA. Differential variability analysis of gene expression and its application to human diseases. Bioinformatics. 2008; 24:i390-8.

20. Eddy JA, Hood L, Price ND, Geman D. Identifying tightly regulated and variably expressed networks by Differential Rank Conservation (DIRAC). PLoS Comput Biol. 2010; 6:e1000792.

21. Buzdin AA, Zhavoronkov AA, Korzinkin MB, Venkova LS, Zenin AA, Smirnov PY, Borisov NM. Oncofinder, a new method for the analysis of intracellular signaling pathway activation using transcriptomic data. Front Genet. 2014; 5:55. 
22. Buzdin AA, Zhavoronkov AA, Korzinkin MB, Roumiantsev SA, Aliper AM, Venkova LS, Smirnov PY, Borisov NM. The OncoFinder algorithm for minimizing the errors introduced by the high-throughput methods of transcriptome analysis. Front Mol Biosci. 2014; 1:8.

23. Borisov NM, Terekhanova NV, Aliper AM, Venkova LS, Smirnov PY, Roumiantsev S, Korzinkin MB, Zhavoronkov AA, Buzdin AA. Signaling pathways activation profiles make better markers of cancer than expression of individual genes. Oncotarget. 2014; 5:10198-205.

24. Lezhnina K, Kovalchuk O, Zhavoronkov AA, Korzinkin MB, Zabolotneva AA, Shegay PV, Sokov DG, Gaifullin NM, Rusakov IG, Aliper AM, Roumiantsev SA, Alekseev BY, Borisov NM, et al. Novel robust biomarkers for human bladder cancer based on activation of intracellular signaling pathways. Oncotarget. 2014; 5:9022-32.

25. Aliper AM, Frieden-Korovkina VP, Buzdin A, Roumiantsev SA, Zhavoronkov A. Interactome analysis of myeloidderived suppressor cells in murine models of colon and breast cancer. Oncotarget. 2014; 5:11345-53.

26. Spirin PV, Lebedev TD, Orlova NN, Gornostaeva AS, Prokofjeva MM, Nikitenko NA, Dmitriev SE, Buzdin AA, Borisov NM, Aliper AM, Garazha AV, Rubtsov PM, Stocking $\mathrm{C}$, et al. Silencing AML1-ETO gene expression leads to simultaneous activation of both pro-apoptotic and proliferation signaling. Leukemia. 2014; 28:2222-8.

27. Aliper AM, Csoka AB, Buzdin A, Jetka T, Roumiantsev S, Moskalev A, Zhavoronkov A. Signaling pathway activation drift during aging: Hutchinson-Gilford Progeria Syndrome fibroblasts are comparable to normal middle-age and oldage cells. Aging (Albany NY). 2015; 7:26-37.

28. Makarev E, Cantor C, Zhavoronkov A, Buzdin A, Aliper A, Csoka AB. Pathway activation profiling reveals new insights into age-related macular degeneration and provides avenues for therapeutic interventions. Aging (Albany NY). 2014; 6:1064-75.

29. Elder D. Tumor progression, early diagnosis and prognosis of melanoma. Acta Oncol. 1999; 38:535-47.

30. Vander Heiden MG, Cantley LC, Thompson CB. Understanding the Warburg effect: the metabolic requirements of cell proliferation. Science. 2009; 324:1029-33.

31. Cantor JR, Sabatini DM. Cancer cell metabolism: one hallmark, many faces. Cancer Discov. 2012; 2:881-98.

32. Ridnour LA, Thomas DD, Donzelli S, Espey MG, Roberts DD, Wink DA, Isenberg JS. The biphasic nature of nitric oxide responses in tumor biology. Antioxid Redox Signal. 2006; 8:1329-37.

33. Roberts DD, Isenberg JS, Ridnour LA, Wink DA. Nitric oxide and its gatekeeper thrombospondin-1 in tumor angiogenesis. Clin Cancer Res. 2007; 13:795-8.

34. Almeida A, Moncada S, Bolaños JP. Nitric oxide switches on glycolysis through the AMP protein kinase and 6-phosphofructo2-kinase pathway. Nat Cell Biol. 2004; 6:45-51.
35. Thomas DD, Espey MG, Ridnour LA, Hofseth LJ, Mancardi D, Harris CC, Wink DA. Hypoxic inducible factor 1alpha, extracellular signal-regulated kinase, and p53 are regulated by distinct threshold concentrations of nitric oxide. Proc Natl Acad Sci USA. 2004; 101:8894-9.

36. Feun L, You M, Wu CJ, Kuo MT, Wangpaichitr M, Spector S, Savaraj N. Arginine deprivation as a targeted therapy for cancer. Current pharmaceutical design. 2008; 14:1049.

37. Yerushalmi HF, Besselsen DG, Ignatenko NA, BlohmMangone KA, Padilla-Torres JL, Stringer DE, Guillen JM, Holubec H, Payne CM, Gerner EW. Role of polyamines in arginine-dependent colon carcinogenesis in Apc(Min) (/+) mice. Mol Carcinog. 2006; 45:764-73.

38. Gilmour SK. Polyamines and nonmelanoma skin cancer. Toxicol Appl Pharmacol. 2007; 224:249-56.

39. Igarashi K, Sakamoto I, Goto N, Kashiwagi K, Honma R, Hirose S. Interaction between polyamines and nucleic acids or phospholipids. Arch Biochem Biophys. 1982; 219:438-43.

40. Gerner EW, Meyskens FL. Polyamines and cancer: old molecules, new understanding. Nat Rev Cancer. 2004; 4:781-92.

41. Di Marino D, D'Annessa I, Tancredi H, Bagni C, Gallicchio E. A unique binding mode of the eukaryotic translation initiation factor $4 \mathrm{E}$ for guiding the design of novel peptide inhibitors. Protein Sci. 2015.

42. Medina MA, Correa-Fiz F, Rodríguez-Caso C, SánchezJiménez F. A comprehensive view of polyamine and histamine metabolism to the light of new technologies. J Cell Mol Med. 2005; 9:854-64.

43. Abildgaard C, Guldberg P. Molecular drivers of cellular metabolic reprogramming in melanoma. Trends in Molecular Medicine. 2015; 21:164-71.

44. van Vlies $\mathrm{N}$, Ofman R, Wanders RJA, Vaz FM. Submitochondrial localization of 6-N-trimethyllysine dioxygenase-implications for carnitine biosynthesis. FEBS J. 2007; 274:5845-51.

45. Monfregola J, Cevenini A, Terracciano A, van Vlies N, Arbucci S, Wanders RJA, D’Urso M, Vaz FM, Ursini MV. Functional analysis of TMLH variants and definition of domains required for catalytic activity and mitochondrial targeting. J Cell Physiol. 2005; 204:839-47.

46. Wei YD, Rannug U, Rannug A. UV-induced CYP1A1 gene expression in human cells is mediated by tryptophan. Chem Biol Interact. 1999; 118:127-40.

47. Niu X, Zhang T, Liao L, Zhou L, Lindner DJ, Zhou M, Rini B, Yan Q, Yang H. The von Hippel-Lindau tumor suppressor protein regulates gene expression and tumor growth through histone demethylase JARID1C. Oncogene. 2012; 31:776-86.

48. Wellmann S, Bettkober M, Zelmer A, Seeger K, Faigle M, Eltzschig HK, Bührer C. Hypoxia upregulates the histone demethylase JMJD1A via HIF-1. Biochemical and Biophysical Research Communications. 2008; 372:892-7. 
49. Levy C, Khaled M, Iliopoulos D, Janas MM, Schubert S, Pinner S, Chen P-H, Li S, Fletcher AL, Yokoyama S, Scott KL, Garraway LA, Song JS, et al. Intronic miR-211 Assumes the Tumor Suppressive Function of Its Host Gene in Melanoma. Molecular Cell. 2010; 40:841-9.

50. Mazar J, Khaitan D, DeBlasio D, Zhong C, Govindarajan SS, Kopanathi S, Zhang S, Ray A, Perera RJ. Epigenetic Regulation of MicroRNA Genes and the Role of miR-34b in Cell Invasion and Motility in Human Melanoma. PLoS ONE. 2011; 6:e24922.
51. Shabalin AA, Tjelmeland H, Fan C, Perou CM, Nobel AB. Merging two gene-expression studies via cross-platform normalization. Bioinformatics. 2008; 24:1154-60. 